\title{
Testing the relations of gelotophobia with humour as a coping strategy, self-ascribed loneliness, reflectivity, attractiveness, self-acceptance, and life expectations
}

\author{
Martin Führ \\ Department of Communication and Psychology, University of Aalborg, Denmark
}

\section{Tracey Platt \& René T. Proyer}

Department of Psychology, University of Zurich, Switzerland. tracey.platt@psychologie.uzh.ch

\begin{abstract}
Gelotophobia (the fear of being laughed at) was studied in a sample of $N=1,322$ Danish adolescents aged 11 to 16. When using a measure of coping humour in three different respects (using humour (1) to overcome uncertainty and stress, (2) in relation to aggression and sexuality, and (3) to get cheered up), it was indicated that the fear of being laughed at existed independently from the use of humour as a coping strategy. It is suggested that interventions targeting the positive use of laughter and humour may have a potential for increasing the well-being of adolescents with high levels of the fear of being laughed at. In single item ratings higher levels of gelotophobia were associated with greater self-ascribed loneliness, lower perceived attractiveness, lower self-acceptance, and rather negative life expectancies. Findings are discussed in the light of current literature and with respect to potential implications for the school life of adolescents.
\end{abstract}

Keywords. adolescents; gelotophobia; coping humour; loneliness; self-acceptance.

\section{Theoretical background of the study: Earlier approaches to gelotophobia}

Humour research has given priority to adult humour during the past decades - for example when studying humour as a coping tool, as indicative of social competence, or as an attitude towards life (see Martin 2007 or Ruch 2008 for an overview). Only recently has interest in humour research increased in studying the aversive effects laughter may have. Ruch \& Proyer 
(2008a) published the first empirical study on the fear of being laughed at (gelotophobia). Gelotophobes particularly fear being laughed at and appearing ridiculous to their social partners. They experience humour as aversive and as a means to put them down (see Ruch \& Proyer 2008a, 2008b or Platt \& Forabosco 2012 for an overview). Except for two studies (Proyer, Neukom et al. 2012; Proyer \& Neukom 2013) there is no study on gelotophobia in children and there are also only two studies on gelotophobia in adolescents (Führ 2010; Proyer et al. 2013). However, there are many open questions on gelotophobia in childhood and adolescence, including its causes and consequences. There is evidence that children use humour as a coping mechanism (Masten 1986), and this ability may contribute positively to avoiding gelotophobia and its detrimental effects. Since most of the studies in the field were conducted with adults, the present state of knowledge in the field clearly warrants a more in depth analysis of the role of gelotophobia in adolescents as well.

Earlier studies pointed at interesting age-related effects. For example, in a study of people more than 50 years old, Platt et al. (2010) showed that various vulnerabilities related to higher age gave gelotophobes further reasons to withdraw, which may have a negative impact for them with respect to them getting support from friends, relatives and caregivers. Platt \& Ruch (2010) studied age related vulnerabilities in more detail and how they were related to three dispositions towards being laughed at, namely gelotophobia, gelotophilia (the joy of being laughed at), and katagelasticism (the joy of laughing at others; see Ruch \& Proyer 2009). These dispositions towards ridicule and being laughed at were introduced to describe potential reactions in laughter-type situations in more detail. In the Platt \& Ruch (2010) study participants were first asked whether they had ever experienced the various age related vulnerabilities, and secondly, whether they worried about them as they got older. The findings suggest that the gelotophiles neither worry nor acknowledge experiencing the identified vulnerabilities. It seems that even if the gelotophiles had experienced vulnerabilities like loneliness, failing health or financial problems, they did not worry about them. Gelotophiles who were more highly educated and who did not worry, also had a tendency to recount their problems to others in order to make them laugh. However, the picture was very different for those with gelotophobia. They were not inclined to tell anyone about their anticipated concerns for fear of ridicule, but they worried about the vulnerabilities, even when they had never experienced them. As regards the vulnerability of loneliness, earlier research on gelotophobia in adults (Ruch 2008) has shown that individuals with high scores on gelotophobia more significantly report the feeling of loneliness. This study based on an adult sample clearly shows that humour and the ability to joke about the weaknesses that come with old age help to facilitate or maintain connections with other people: being able to turn difficult conditions of life into something to laugh about, makes a person less likely to worry and to withdraw from the much needed support of others. There is also empirical data which supports the notion that gelotophobia is associated with lower remembered social support in childhood and adolescence from different groups (peers, family, teachers; Weibel \& Proyer 2012). The studies by Platt and colleagues on age-related transgressions in gelotophobia also show clearly that people think that they were more vulnerable to being laughed at and retrospectively indicated greater fear of being laughed at with younger age. This finding is an indicator that it makes sense to study gelotophobia in younger age groups as well as in adulthood.

When thinking about possibilities on how to cope with adversities, humour could be a potent strategy. In fact, Titze (2009) suggests the use of humour drama as one intervention to address gelotophobia in his patients. This might be hindered by a specific stance of gelotophobes towards humour. Ruch et al. (2009) found the fear of being laughed at in adults to be related negatively with cheerfulness and gelotophobes seem to describe their humour styles as inept, socially cold, and mean-spirited. Additionally, they do not endorse self- 
enhancing and social humour. Nevertheless, the ability to create humour existed independently of individual levels of gelotophobia. In this study a measure for coping humour was also used (i.e. to make use of humour in coping with stressful events that people encounter in their lives; Martin \& Lefcourt 1983). As expected, gelotophobia correlated negatively with coping humour $(r=-.37, p<.001, N=112)$. This confirms early theoretical views on gelotophobia (see Ruch \& Proyer 2008a; Titze 2009), according to which gelotophobes do not experience humour and laughter as relaxing but more as aversive and, of course, this is associated with an inability to use humour as a means of coping with adversities. Only these few studies have been conducted on the impact of gelotophobia on these matters in adults, and so far nothing is known whether the ability to use humour as a coping tool when being laughed at can protect a person from feeling low. However, it seems reasonable to suggest that children who are able to laugh at themselves in various situations may be less vulnerable to being the subject of others' laughter. In line with Ruch et al. (2009), we expect that findings for adolescents will be similar; i.e. a negative relation between gelotophobia and self-ascribed use of humour for coping with adversities.

Some earlier studies on much younger samples highlight the importance of humour as a social and communicative competence and as an attitude towards life in early adolescence. As to the view of humour as an attitude towards life, prominent early researchers like Høffding (1916) and Freud (1905) argue that children lack both the necessary life experience and the cognitive preconditions. However, Führ (2002) shows in a study of 962 Danish children aged 11-16 years that there is reason to believe that humour as an attitude towards life can be found in that age group.

Führ (2002) showed that children aged 11-16 with high humorous coping scores reported to be less lonely and had an overall more positive view of their own personality. The majority of studies have focused on adults, but several important findings (e.g. Führ 2002), indicate that children with a well developed sense of humour show greater self-confidence, are more popular, less lonely and find it easier to establish positive relations and friendship with their peers. Furthermore, the ability to use humour in various daily life situations appears to be a strong predictor for happiness and wellbeing.

Classical developmental psychology (e.g. Erikson 1968) regards adolescence as the most difficult period in human life. The challenge of growing sexual maturity indicated by visible changes of the body, sexual interest in one's own and inter-gender sexuality, and the responses of the surroundings to these changes, as well as the growing ego-reflectivity described by Stern (1925) has to be considered as a common challenge for this age group. In spite of many differences between divergent scientific approaches to the question of how humour develops in childhood, there is an overall agreement about the fact that children, as well as adults, use humour to tackle actual life tasks. It needs to be mentioned that these changes at so many different levels, of course, also can be seen as risk factors increasing the chance of being laughed at (e.g. a sudden change of the tone of the voice in a conversation, or clumsy movements resulting from growth processes, etc.). Hence, not only the question of how humour can be used for dealing with adversities but also how to deal with ridicule and laughter are important issues at this age (see Führ 2010; Proyer et al. 2013).

Furthermore, the questions "who am I" and "which way do I want to go" is redefined now including the challenge to move from the dependence on family towards the first own attempt of an attitude towards life. Kauke (1996) refers to this as Lebensentwurf (outcast of life), an outline for a wished self, which is seen as an aim for the development of self. Transition from childhood to adolescence is seen as a step towards personal awareness, implying self-observation and self-reflexivity. The growing awareness of one's own self maintains a conflict potential, wherever the expectations of, e.g., the family, friends and surroundings differ from the child's first personal project (Kauke 1996). Furthermore, 
substantial choices have to be made, like the choice of school type, subjects or choices combined with other educational matters, following the first attempt towards an outlook of life. Thus, adolescence generally appears to be a very difficult and vulnerable period in childhood. As mentioned earlier, this vulnerability may open the door for gelotophobia (the fear of being laughed at). The child's various attempts to test different approaches to form one's own outlook of life (e.g. new clothing to signal sympathy or affiliation to a group of peers, or inclinations to share specific values or hobbies) may not always receive a positive reaction. However, adolescents deal differently with these incidents and it is yet unknown what factors can contribute to individual differences in gelotophobia. Social support seems to play a role (Weibel \& Proyer 2012). Additionally, studies suggest that gelotophobia is uncorrelated if measured in children between seven to eight years old and their parents (Proyer \& Neukom 2013), but is positively correlated if measured in adult children (all $\geq 18$ years of age) and their parents ( $r=.40, p<.01, N=85$; Proyer, Estoppey et al. 2012). In late adolescents it seems as if the children model their reaction towards ridicule and being laughed at after their parents. One might argue that this could be helpful for dealing with the stress caused by laughter and being laughed at and could be interpreted in the sense of a coping mechanism, which may protect children from further harm (for an overview on familial aggregations in dealing with humour, see Manke 1998). Additionally, it was found that parenting styles had an impact on the expression of gelotophobia in adult children. The remembered parenting styles were assessed in the children but also in the parents. Gelotophobia in the adult children correlated positively with the usage of rejection and punishment (e.g. punishing the child even for minor things; physical punishment; or eliciting shame in the children) and control and over-protection (e.g. worrying that the child might be harmed; not accepting the friends that the child meets; or push the child to become 'the best'); this was found in the memories of the children and the parents. Additionally, the children also remembered lower warmth (e.g. being low in showing the child ones love; supporting the child; or cuddling the child). Hence, the parenting styles also seem to contribute to the expression of gelotophobia.

A first indicator of the importance of gelotophobia in adolescence was provided by Führ (2010). In a large sample $(N=1,322)$ of Danish school pupils aged 11-16 years, the participants answered the GELOPH $\langle 15\rangle$, which is a self report questionnaire measuring the fear of being laughed at (Ruch \& Proyer 2008b). Whereas the result of an earlier study of Danish adults (Führ et al. 2009) showed that less than $2 \%$ in the adult sample fulfilled the criteria for being slight gelotophobic, the result for the 2010 study was that about 7 times as many adolescents $(14.67 \%)$ fulfilled the criteria. However, this needs to be interpreted conservatively, since the cut-off scores for the GELOPH $<15\rangle$ were derived for adult samples and, therefore, caution is warranted if interpreting these scores in non-adult samples. Führ (2010) found that the GELOPH <15> showed good psychometric properties and revealed a one-factor structure in the sample of adolescents. Therefore, Führ concluded that the scale (the original adult version) can also be used with adolescents. In any case it is clear that the fear of being laughed at turned out to be a relevant factor in the adolescents' attempts at "finding" their identity and having their first outlook towards adult life. In a similar line, Proyer et al. (2013) recently applied the adult version of the PhoPhiKat-45 (Ruch \& Proyer 2009; measuring gelotophobia, gelotophilia, and katagelasticism) to a sample of 13 to 15year-old Swiss adolescents. As in Führ (2010), it was shown that the prevalence of gelotophobia was higher in the adolescents than in the adults. Additionally, gelotophobia was associated with experiences of being a victim of bullying and katagelasticism increased with ratings of being a bully. These findings were stable for self- but also peer-reports from classmates. Overall, this highlights the relevance of gelotophobia in a school context and 
potential practical applications (e.g. bullying prevention programmes also considering the role of laughing and laughing at).

\section{The aim of the present study}

The main aim of the present study is to investigate the relationship between gelotophobia and the use of humour for coping against various adversities among adolescents. Coping in three areas will be considered, namely coping with uncertainty and stress, coping with aggression and sexuality and the use of humour to get cheered up/to cheer others up. Analyses will be conducted for boys and girls separately, and differences between age groups will also be considered. It was expected that gelotophobia is negatively associated with using humour as a coping strategy (cf. Ruch et al. 2009). A further aim of this study is learning more about the correlates of gelotophobia in adolescents. Therefore, gelotophobia will be studied in relation to the presumed self-ascribed loneliness, reflectivity, perceived attractiveness, selfacceptance, and life expectations. These analyses are rather of an exploratory nature. However, earlier studies showed that gelotophobes tend to underestimate themselves; e.g. their humour (Ruch et al. 2009), their intelligence (Proyer \& Ruch 2009b), or their virtuousness (Proyer \& Ruch 2009a; Proyer et al. 2013). Additionally, gelotophobia in adults is negatively associated with life satisfaction (Proyer, Ruch et al. 2012; Weibel \& Proyer 2012) and optimism (Proyer \& Ruch 2009a; Proyer et al. 2014). It was expected that this rather negative view of oneself permeates into lower ratings regarding all positive traits or life expectancies. These correlations will help for a better understanding of gelotophobia in a variety of variables relevant to adolescents.

\section{Method}

\subsection{Sample}

A total of $N=1,322$ adolescents between 11 and 16 years participated in this study $(\mathrm{M}=13.5$ years). Table 1 gives a breakdown of sample characteristics (age and gender distribution). The adolescents were in one of eleven schools with 91 classes in grades 5 to 9 .

Table 1. Age and Gender Distribution in the Sample $(\mathrm{N}=1,322)$

\begin{tabular}{|c|c|c|c|c|}
\hline Age & Boys & Girls & Unknown & Total \\
\hline 11 years & 53 & 46 & 3 & 102 \\
\hline 12 years & 124 & 123 & 8 & 255 \\
\hline 13 years & 139 & 148 & 4 & 291 \\
\hline 14 years & 160 & 169 & 8 & 337 \\
\hline 15 years & 118 & 110 & 3 & 231 \\
\hline 16 years & 59 & 43 & 4 & 106 \\
\hline Total & 653 & 639 & 30 & 1322 \\
\hline
\end{tabular}




\subsection{Instruments}

The GELOPH $<15>$ (Ruch \& Proyer 2008b) is a 15-item questionnaire for the subjective assessment of gelotophobia. Führ et al. (2009) authored the Danish version, which was used in the present study. All items are positively keyed and the 4-point answer format ranges from 1 = "strongly disagree" to $4=$ "strongly agree." The GELOPH $<15>$ is the standard instrument for the assessment of the fear of being laughed at. It has been widely used in research and has so far been translated to more than 40 different languages (e.g. Edwards et al. 2010; Papousek et al. 2009; Platt et al. 2009; Ruch et al. 2009; Ruch \& Proyer 2009a, 2009b). The alpha-coefficient in this sample was .84 .

The Children Coping Humour Strategy Survey (CCHSS; Führ 2002) is an 11-item questionnaire developed to investigate children's use of humour when tackling various challenges in daily life (for a closer description of the CCHSS, see Führ 2002). The CCHSS showed good psychometric properties in Führ (2002), and factor analysis pointed at a three factor solution; i.e. the use of humour when tackling, a) uncertainty and stress; b) aggression and sexuality; and c) the use of humour to get cheered up/to cheer others up. The 6-point answer format ranges from $1=$ "strongly disagree" to $6=$ "strongly agree". Alpha-coefficients in this sample were between .73 and .75 .

Participants completed single item ratings on whether they feel lonely, whether they think of themselves as a reflective person, on their self-acceptance, and on their level of positive life expectancy on a 6-point scale ( $1=$ "not at all" to $6=$ "to the highest degree"); and on their perceived attractivity on a 4-point scale ( $1=$ "not at all" to $4=$ "very much").

\subsection{Procedure}

As humour, especially as a communicative, social and coping tool in children has been the subject of massive public interest in recent years, several articles and interviews on this matter were available in schools in Denmark, which helped generating the data for this study. The majority of the schools participating in this study reacted on a request by the first author, when announcing the wish to investigate humour in childhood and adding the question of gelotophobia in children to earlier research subjects.

As a first step the community school boards received broad informational material about the background, subject and purpose of the investigation. After agreeing in principle to the research project, each school was contacted separately. All teachers involved received instructions about how to carry out the survey. It appeared doubtful at the beginning of the project whether the youngest participants in grade 5 (aged approximately 11 years) would be able to read and understand all questions by themselves. Therefore, the teachers were asked to read each question and be of assistance if necessary in the fifth grade (e.g. answering questions upon request; explaining specific words, etc.), whereas all other grades completed the questionnaire handed out by their teachers. Parents had to provide consent for the participation of their children, and the information on absences was handed out to the project leader by the school administration. The participants were informed that participation in the study was voluntary and that they could refuse participation or stop filling in the questionnaires at any time they wanted to.

The survey covers geographically all parts of Denmark, with participating schools from the very south of Jutland and the north of Sealand including the islands of Falster and Lolland. Demographically the study covers urban and town schools, a catholic private school, high school in Copenhagen, as well as an urban school with $35 \%$ of non-ethnic Danes. All information provided by the school as well as the questionnaire data could be treated 
completely confidentially and correlated to each other due to the code-system applied, but as a duty of care to minors, all questionnaires could be traced back to the individual participant.

\section{Results}

\subsection{The relations between gelotophobia and humour as coping}

Preliminary analyses suggested that all scales were normally distributed and alphacoefficients were comparable in all age groups. The total score of the GELOPH $<15>$ (Führ et al. 2009) was correlated with the Children Coping Humour Strategy Survey (CCHSS; Führ 2002). Correlations (Pearson) were computed for the three subscales (a) coping with uncertainty and stress; (b) coping with aggression and sexuality; and (c) the use of humour to get cheered up/to cheer others up. Data were analysed for the total sample but also separately for boys and girls; the coefficients are given in Table 2:

Table 2. Correlation Coefficients between Gelotophobia and Components of the Children Coping Humour Strategy Survey.

\begin{tabular}{llllllllll}
\hline & All & \multicolumn{3}{c}{ Boys } & \multicolumn{3}{c}{ Girl } \\
\cline { 2 - 9 } & Stress & Agg & Cheer & Stress & Agg & Cheer & Stress & Agg & Cheer \\
\hline Total & .01 & $.10^{* *}$ & -.04 & .05 & .03 & -.01 & -.01 & $.17^{* *}$ & -.08 \\
$11-\mathrm{y}$ & .08 & .18 & -.16 & .06 & .11 & $-.29 *$ & .14 & $.37^{*}$ & -.01 \\
$12-\mathrm{y}$ & $.24 * *$ & $.21^{* *}$ & .04 & $.28^{* *}$ & .11 & .14 & $.21^{*}$ & $.28^{*}$ & -.04 \\
$13-\mathrm{y}$ & .05 & $.14^{*}$ & -.03 & .09 & .13 & -.02 & .01 & .14 & -.04 \\
$14-\mathrm{y}$ & $-.14 *$ & .03 & $-.12^{*}$ & -.09 & .02 & -.03 & -.15 & .05 & $-.20^{* *}$ \\
$15-\mathrm{y}$ & .01 & .12 & -.04 & .00 & -.01 & -.05 & .02 & $.26^{* *}$ & -.05 \\
$16-\mathrm{y}$ & -.09 & -.05 & .09 & .05 & -.19 & .09 & -.14 & .17 & .08
\end{tabular}

Note. $N($ total $)=1294-1322(643-653$ boys; $624-639$ girls $) ; n(11$ year $)=98-102(52-53$ boys; $44-46$ girls $) ; n(12$ year $)=250-255$ (124 boys; $119-123$ girls $) ; n(13$ year $)=284-291$ (136-139 boys; $144-148$ girls); $n$ (14 year) $=329-337$ (157-160 boys; $164-169$ girls); $n(15$ year $)=229-231$ (117-118 boys; 110 girls); $n(16 y e a r)=104-106$ (57-59boys; 43 girls); Stress = uncertainty and stress; Agg = aggression and sexuality; Cheer $=$ the use of humour to get cheered up/to cheer others up.

$* p<.05 ; * * p<.01$.

Table 2 shows that gelotophobia had a mean score of 1.86, and a standard deviation of .53 which is comparable to Führ (2010) that had a mean score of 1.94 and standard deviation of .52 existed widely independently from components of children's use of humour for coping. There was a small positive relation with aggression and sexuality $\left(r^{2}=.01\right)$. If boys and girls are considered separately, there was no correlation for the boys between gelotophobia and coping with aggression, whereas the correlation coefficient was $.17\left(r^{2}=.03\right)$ for the girls. If the age groups are analysed separately, there were moderate positive relations between uncertainty and stress and gelotophobia (in boys and girls) and aggression and sexuality in the girls. It was striking that several correlation coefficients were negative for the 14-year-old pupils. Inclination to use humour to cope with aggression and sexuality was positively correlated with gelotophobia in the 12-year-old pupils as well as and in 11- and 15-year-old girls. Furthermore, there was a negative relation between greater fear of being laughed at and the use of humour to get cheered up/to cheer others up in the 11-year-old boys and in 14- 
year-old girls. The multiple squared correlation coefficient between gelotophobia and all three scales of the CCHSS was $R^{2}=.02$ indicating independence of the two measures.

\subsection{Relations between gelotophobia and various self-descriptions}

The adolescents answered questions about (a) how lonely they perceived themselves, (b) whether they considered themselves as a rather reflective person or not, (c) whether they perceived themselves as attractive, (d) their self-acceptance, and (e) whether they have a positive expectation towards their own life (cf. Führ 2010). The adolescents provided single item ratings (six-point scale). The frequency distribution of loneliness-ratings is shown separately in Figure 1.

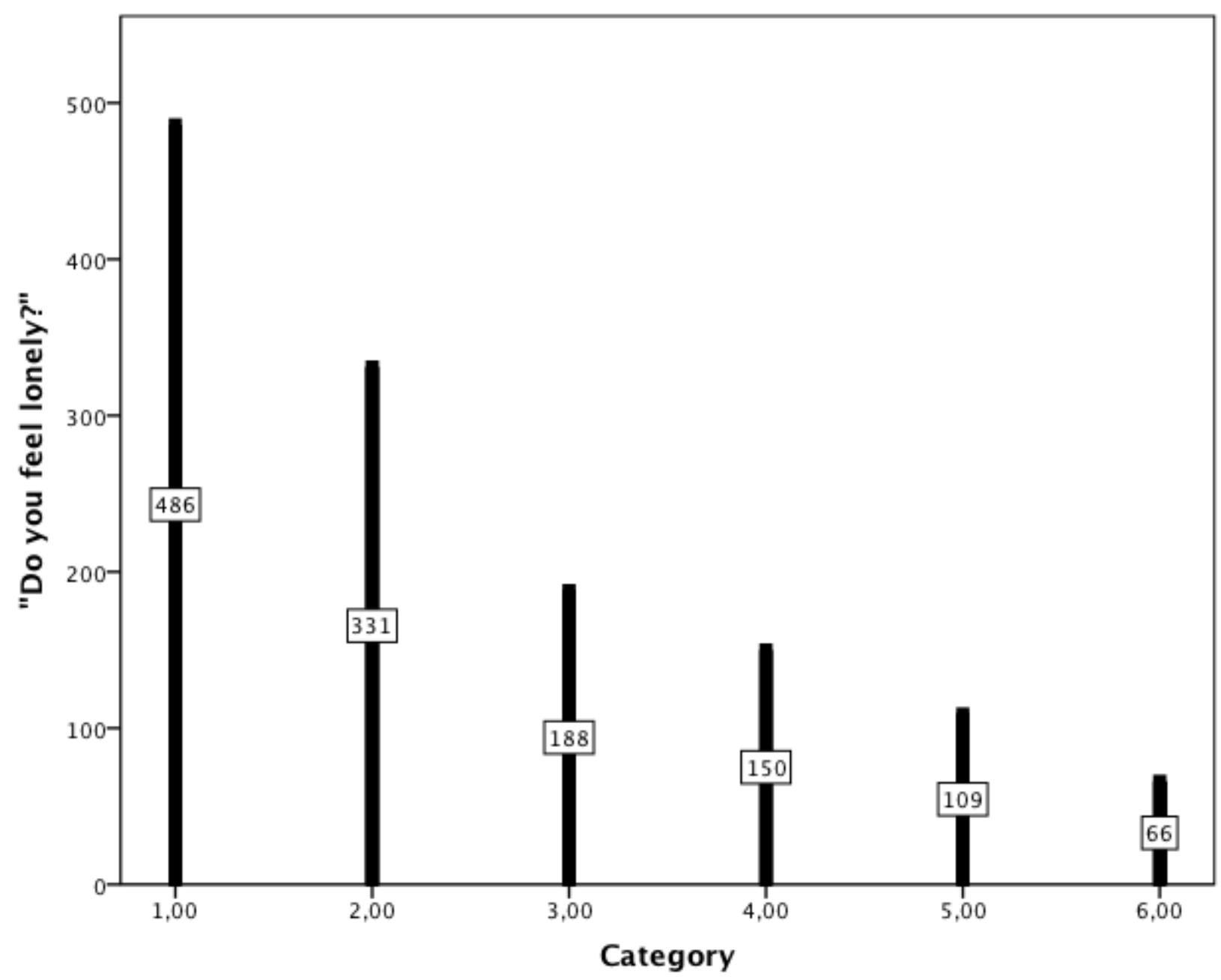

Figure 1. Frequency of ratings for loneliness in children aged 11-16 years. Categories are from $1=$ "not at all lonely" to $6=$ "to the highest degree of loneliness."

Figure 1 shows that there were 486 adolescents who did not feel lonely at all, followed by $n=$ $331, n=188, n=150, n=109$, and $n=66$ in the following categories ( $M=2.45$ on a 6-point scale; median =2). Of those feeling lonely to the highest degree, there were 40 girls and 26 boys. Overall, girls $(\mathrm{M}=2.25, \mathrm{SD}=1.45)$ scored higher than boys $(\mathrm{M}=2.62, \mathrm{SD}=1.54)$ in their perceived loneliness, $t(1296)=4.85, p<.001$. The scale for the reflectivity had a mean score of 4.09 (median $=4$ ) and less than five percent indicated that they were not reflective at 
all. Girls $(\mathrm{M}=4.39, \mathrm{SD}=1.28)$ described themselves with greater self-reflection than the boys $(\mathrm{M}=3.82, \mathrm{SD}=1.44), t(1298)=-7.78, p<.001$. About $6 \%(n=88)$ indicated that they were not good looking at all $(M=4.24$, median $=4)$ but more than one fifth $(22.8 \%)$ rated themselves as very good-looking. Boys $(\mathrm{M}=3.80, \mathrm{SD}=1.32)$ rated their attractiveness higher than the girls $(\mathrm{M}=3.66, \mathrm{SD}=1.38) \mathrm{did}, t(1298)=4.25, p<.001$. The self-acceptance scale had a mean score of 4.48 (median $=5$ ) and about one third $(30.3 \%$ ) was very happy with the way they were. Boys were also higher in their self-acceptance ratings than the girls, $t(1297)=4.34, p<.001$. About $2.7 \%$ of the children had not at all a positive outlook on their lives while close to one half $(48.5 \%)$ had a highly positive outlook on their life. The mean score was 3.32 (median $=3$; 4-point rating for this item). Boys indicated having a more positive life expectations than the girls did, $t(1264)=2.53, p<.05$. The self-ratings were correlated with the gelotophobia scores; the coefficients are given in Table 3.

Table 3. Correlations between Gelotophobia and Self-Ratings of Loneliness, Reflectivity, Appearance Towards Others, Self-Acceptance, and Positive Life Expectation

\begin{tabular}{|c|c|c|c|}
\hline & & Boys & Girls \\
\hline Loneliness & $.35 * *$ & $.32 * *$ & $.36^{* *}$ \\
\hline Reflectivity & $.12 *$ & $.09 *$ & $.13 * *$ \\
\hline Attractiveness & $-.25 * *$ & $-.22 * *$ & $-.28 * *$ \\
\hline Self-acceptance & $-.26 * *$ & $-.23 * *$ & $-.28 * *$ \\
\hline Positive expectation & $-.21 * *$ & $-.19 * *$ & $-.23 * *$ \\
\hline
\end{tabular}

Note. $N=1,266-1,331 ; N($ Boys $)=638-658 ; N($ Girls $)=624-640$. Attractive $=$ Attractiveness; Positive expectation $=$ Positive life expectation.

$* p<.05 ; * * p<.01$.

Table 3 shows that gelotophobia was positively correlated with self-ratings of loneliness (in boys and girls to the same degree) with an $r^{2}$ of about .12. There was a numerically small but significant positive association between the fear of being laughed at and greater selfreflectivity. The size of the correlation coefficients, however, was rather small and perhaps practically negligible. Those with greater fear of being laughed at also described themselves with lower attractiveness, lower self-acceptance, and not with positive expectations towards their lives. When different age groups were analysed separately, the findings were rather similar, and therefore they are not reported in detail.

\section{Discussion}

This is the first study of the relation between gelotophobia and the use of humour for coping with adversity in adolescents. The findings suggest that the fear of being laughed at exists widely independently from the self-ascribed use of humour as a coping strategy. The zerocorrelations can be interpreted in the sense that there are gelotophobes who are able as well as others who are unable to use humour as a coping strategy. This was surprising insofar as findings with adults indicated a negative relation between gelotophobia and the use of coping humour (Ruch et al. 2009). One might argue that adolescents are still in a kind of "orientation"-phase and need to learn how to properly deal with humour and laughter-and, especially, the usage of humour to cope with adversities. It has been argued earlier that adolescence is a period of transition. These changes bring many opportunities for being laughed at and put the adolescents at higher risk of being laughed at. It would be interesting to study those gelotophobic adolescents, who can use humour to cope with stressors in 
comparison to those, who cannot use humorous coping. Ruch \& Proyer (2008a) proposed the differentiation between "pure" and "real" gelotophobes with the difference being that the real gelotophobes get laughed at and fear what they experience while the pure gelotophobes fear imagined ridicule and do not get laughed at by others. It would be interesting to see whether this differentiation has links with the usage of humorous coping as well. Currently this is at the level of speculation and needs further investigation. However, it seems reasonable that adults and adolescents seem to differ in the respect to the interplay between gelotophobia and using humour to cope with adversities. If frequent and repeated but most importantly intense experiences of being laughed at (see Edwards et al. 2010; Proyer et al. 2009; Ruch \& Proyer 2008a; Titze 2009) are linked to the individual expression of gelotophobia, one might argue that adults have had more possibilities of experiencing such situations and incidents and, therefore, may have lost the capacity to use humour for coping. Or, it may be that, as Platt \& Ruch (2010) indicated, more people remember a time around their teenage years where they were gelotophobic. It is possible that those still having humour to cope are those that do eventually grow out of it, as the positive side of using humour to cope gets reinforced over time. Unfortunately, there are no longitudinal data on gelotophobia yet, so any developmental processes are at the level of speculation only.

All data collected so far shows higher levels of gelotophobia in younger samples than in adults; this has been shown for children from the age of 6 put to adolescents. In earlier work, we have argued that this may be attributed to specifics of the situation, i.e. being in school and having to frequently interact with others, but also learning the differences between laughing with and laughing at. We do not think that the sheer number of gelotophobes plays a role for the relationship with coping humour. It seems evident, however, that learning to use humour as a coping tool seems perhaps to be even more important at a younger age than at an older age. As said, this is presumably the time when children learn to use humour and laughter appropriately-with varying success though. Using a sample of 112 students, Ruch et al. (2009) found a robust negative relationship of coping humour (Martin \& Lefcourt's 1983 scale) with gelotophobia $(r=-.37, \mathrm{p}<.001)$. Apparently gelotophobes have difficulties using humour to cope with adversities - at both, younger and higher ages.

It was evident that gelotophobia was associated with feelings of loneliness in both boys and girls. Of course, the present study does not allow for causal inferences; i.e. it cannot be implied whether adolescents are gelotophobic because they are lonely (e.g. lacking opportunities to practise social skills associated with laughter and humour in general), or whether they are lonely because they are gelotophobic (e.g. being perceived as somewhat "strange" by classmates and peers). Nevertheless the relationship can have an impact on school life. Führ (2010) has already shown that gelotophobia is associated with more frequent thoughts about skipping school (without actually having a higher number of absent days), and a study by Edwards et al. (2010) suggests that subjects with high gelotophobia scores remember having been laughed at in school in spite of academic success; they also report to have been laughed at by peers as well as teachers (Ruch et al. 2010). In any case it seems as if the perception of lacking social support has an impact on the fear of being laughed at (Weibel \& Proyer 2012).

There were small positive correlations between gelotophobia and how reflective the adolescents perceived themselves. However, at the moment it is unclear how to interpret this fact. One way would be to argue that children who were laughed at in the past ponder about it and reflect on what the reasons might have been. In fact, a hierarchical factor analysis of the GELOPH <15> in extreme scorers in gelotophobia yields that there are three positively correlated components of coping with derision (by control, withdrawal, internalising), disproportionate negative responses to being laughed at, and paranoid sensitivity to anticipated ridicule (Platt et al. 2012). It might be plausible to assume that the reflectivity is 
part of coping with derision: reflective people will ponder on why others laugh at them as part of the internalisation of the belief that they are indeed someone to be laughed at.

It was evident that there is a tendency for those with high gelotophobia scores to perceive themselves as rather not-attractive. So far, no data is available that shows whether gelotophobes differ from non-gelotophobes in their physical appearance, or whether others perceive them as more or less attractive. However, it should be reminded that gelotophobes do underestimate their intelligence as well as their humour production abilities (Proyer \& Ruch 2009a; Ruch et al. 2009). Neither is data available on how gelotophobes behave in intimate relationships; one study by Proyer, Estoppey et al. (2012) indicates that gelotophobes seem to stick together as there were positive relations between the gelotophobia scores of couples (see also Proyer \& Neukom 2013). Platt \& Forabosco (2012) find that adult gelotophobes are more often single; even at an older age they relatively often stay in their parents' household.

Gelotophobia was also associated with lower levels of self-acceptance. This seems in line with expectations derived from descriptions of the experiential world of gelotophobes (e.g. Ruch \& Proyer 2008; Titze 2009) and lowered perception of their own abilities and strengths (Proyer \& Ruch 2009a, 2009b; Proyer et al. 2013). This might be a valuable starting point for the development of intervention programmes for gelotophobia. Of course, the direction of causality cannot be determined form this data but it seems to be a reasonable assumption that people with higher self-acceptance have more confidence not to appear as ridiculous to their social interaction partners (cf. Ruch \& Proyer 2008a; Titze 2009). Hence, programmes addressing self-acceptance may be helpful.

Finally, gelotophobia was negatively associated with positive life expectations which is in line with earlier findings of lower levels of life satisfaction in gelotophobes (Proyer, Ruch et al. 2012; Weibel \& Proyer 2012) as well as with data showing a negative correlation between gelotophobia and optimism as a strength of character (Proyer \& Ruch 2009b; Proyer et al. 2014). Correlation coefficients for boys and girls did not differ from each other. Generally, on the basis of these self-ratings gelotophobic adolescents can be described as feeling lonely, with the impression of not being attractive, low self-acceptance, and a negative expectation for their own life. Taken together findings on greater levels of bullying experience with increasing levels of gelotophobia in children (Proyer, Neukom et al. 2012), adolescents (Führ 2010; Proyer et al. 2013), and adults (Platt et al. 2009), this seems to point the need of addressing the question of laughter, ridicule and being laughed at in school programmes. The prior findings for humour as a coping strategy might indicate that this could be a resource which can be addressed in intervention programmes (see also Titze 2009). One way to facilitate changes might be not to focus on laughter but rather on implementation of positive emotions in gelotophobes. A recent experiment with adults showed that gelotophobes generally display genuine smiles and laughter less frequently and less intensively (Platt et al. 2013). In this study 16 variants of enjoyable emotions were studied, and the Duchenne Display (i.e. the facial expression of joy) was recorded while people talked about emotional experiences. Interestingly, the gelotophobes primarily differed from non-gelotophobes only in those of the 16 enjoyable emotions that typically go along with laughter. However, programmes aimed at bettering the life of gelotophobic children, through positive experiences, need to consider the problem that perhaps such enjoyable emotions that typically do not involve laughter (e.g. gratitude, contentment) would be least aversive. A recent finding in adult individuals shows that they mimicked contempt and not expressions of joy (smiles) compared to those in a no-fear group, and also rated joy in photos displaying faces showing contempt, even though they rated the same amount of contempt as subjects without fear of being laughed at in contempt facial expressions (Hofmann et al. in press). This indicates a misattribution of the expression of enjoyment in others. As contempt 
relates to the expression of moral judgment, this may help us to understand why gelotophobes feel laughed at when they have not been. Training children to understand facial expressions and the emotions that go along with the display of faces may help them form better friendships and thus feel less isolated and lonely.

The study has several limitations. All data was self-reported and may, therefore, be biased in a socially desirable way. Therefore, a replication of the findings (also using different assessment methods; e.g. peer-reports) seems warranted. The comparison of different age groups is purely cross-sectional and developmental processes need rather to be studied using longitudinal designs. Studies developing and testing special forms of gelotophobia items as well as the full PhoPhiKat-45 (Ruch \& Proyer 2009) enable such studies in the future (Führ 2010; Proyer et al. 2013; Proyer, Neukom et al. 2012). Still more studies are needed on the dispositions towards ridicule and being laughed at among children and adolescents as the knowledge on its role in these age groups is rather limited at the moment. A further limitation is that all findings except for those on coping humour are based on single item ratings. Hence, these findings should be regarded as preliminary and should be substantiated with more comprehensive measures and methods (e.g. peer-reports).

This study contributes to understanding of how adolescents deal with ridicule and being laughed at and what role humour for coping with adversities may play in this respect. Findings on coping humour were different from those reported for adults and this can be seen as a call for further and more in depth research in this area. Further analyses provided data which point primarily towards lower self-ascribed attractiveness, self-acceptance and lowered positive life expectations associated with greater levels of gelotophobia, which fits rather well to expectations derived from studies on adult samples.

\section{References}

Edwards, K. R., Martin, R. A., \& Dozois, D. J. A. (2010). 'The fear of being laughed at, social anxiety, and memories of being teased during childhood'. Psychological Test and Assessment Modelling 52, pp. 94-107.

Erikson, E. H. (1968). Identity: Youth and crisis No. 7. WW Norton \& Company.

Freud, S. (1905). Der Witz und seine Beziehungen zum Unbewussten. [Jokes and Their Relation to the Unconscious]. Frankfurt am Main: S. Fischer Verlag.

Führ, M. (2002). 'Coping humour in early adolescence'. Humour: International Journal of Humour Research 15, pp. 283-304.

Führ, M. (2010). 'The applicability of the GELOPH<15> in children and adolescents: First evaluation in a large sample of Danish pupils'. Psychological Test and Assessment Modeling 52, pp. 60-76.

Führ, M., Proyer, R. T. \& Ruch, W. (2009). 'Assessing the fear of being laughed at (gelotophobia): First evaluation of the Danish GELOPH<15>'. Nordic Psychology 61, pp. 62-73.

Hoffding, H. (1916). Den store Humour. En psykologisk stadie. Kobenhavn og Kris-tiania: Gyldendalske Boghandel. Nordisk Forlag.

Hofmann, J., Platt, T., Ruch, W. \& Proyer, R. T. (2015). 'Individual differences in the decoding and responding to joy and contempt'. URL: http://sgo.sagepub.com/content/5/2/2158244015581191. full-text.pdf+html

Kauke, M. (1996). 'Are children still having fun? Observations on humour in schoolchildren'. Gruppendynamik-Zeitschrift fur Angewandte Socialpsychologie 27, pp. 399-414. 
Manke, B. (1998). 'Genetic and environmental contributions to children's interpersonal humour', in Ruch, W. (ed.), The Sense of Humour: Explorations of a Personality Characteristic, Berlin: Mouton de Gruyter, pp. 361-384.

Martin, R. A. (2007). The Psychology of Humour: An Integrative Approach. Burlington, MA: Elsevier Academic Press.

Martin, R. A. \& Lefcourt, H. M. (1983). 'Sense of humour as a moderator of the relation between stressors and moods'. Journal of Personality and Social Psychology 45, p. 1313.

Masten, A. S. (1986). 'Humour and competence in school-aged children'. Child Development 57, pp. 461-473.

Papousek, I., Ruch, W., Freudenthaler, H. H., Kogler, E., Lang, B. \& Schulter, G. (2009). 'Gelotophobia, emotion-related skills and responses to the affective states of others'. Personality and Individual Differences 47, pp. 58-63.

Platt, T. \& Forabosco, G. (2012). 'Gelotophobia: The fear of being laughed at', in Gremigni, P. (ed.), Humour and Health Promotion, Hauppauge, NY: Nova Science Publishers, pp. 229-252.

Platt, T., Hofmann, J., Ruch, W. \& Proyer, R. T. (2013). 'Duchenne Display responses towards sixteen enjoyable emotions: Individual differences between no and fear of being laughed at'. Motivation and Emotion 37, pp. 776-786.

Platt, T., Proyer, R.T., \& Ruch, W. (2009). 'Gelotophobia and bullying: The assessment of the fear of being laughed at and its application among bullying victims'. Psychology Science Quarterly 52, pp. 135-137.

Platt, T. \& Ruch, W. (2010). 'Gelotophobia and age: Do dispositions towards ridicule and being laughed at predict coping with age-related vulnerabilities?'. Psychological Test and Assessment Modeling 52, pp. 231-244.

Platt, T., Ruch, W., Hofmann, J. \& Proyer, R. T. (2012). 'Extreme fear of being laughed at: Components of gelotophobia and the inability to distinguish between ridicule and teasing'. Israeli Journal of Humor Research 1, pp. 86-106.

Platt, T., Ruch, W. \& Proyer, R. T. (2010). 'A lifetime of the fear of being laughed at: An aged perspective'. Zeitschrift für Gerontologie und Geriatrie 43, pp. 36-41.

Proyer, R. T., Estoppey, S., \& Ruch, W. (2012). 'An initial study on how families deal with ridicule and being laughed at: Parenting styles and parent-child-relations with respect to gelotophobia, gelotophilia, and katagelasticism'. Journal of Adult Development 19, pp. 228-237.

Proyer, R. T., Hempelmann, C. F., \& Ruch, W. (2009). 'Were they really laughed at? That much? Gelotophobes and their history of perceived derisibility'. Humour: International Journal of Humour Research 22, pp. 213-231.

Proyer, R. T., Meier, L., Platt, T. \& Ruch, W. (2013). 'Dealing with laughter and ridicule in adolescence: Relations with bullying and emotional responses'. Social Psychology of Education: An International Journal 16, pp. 399-420.

Proyer, R. T. \& Neukom, M. (2013). 'Ridicule and being laughed at in the family: Gelotophobia, gelotophilia, and katagelasticism in young children and their parents'. International Journal of Psychology 48, pp. 1191-1195.

Proyer, R. T., Neukom, M., Platt, T. \& Ruch, W. (2012). 'Assessing gelotophobia, gelotophilia, and katagelasticism in children: An initial study on how six to nine-year-olds deal with laughter and ridicule and how this relates to bullying and victimisation'. Child Indicators Research 5, pp. 297-316.

Proyer, R. T., \& Ruch, W. (2009a). 'How virtuous are gelotophobes? Self- and Peer-reported character strengths among those who fear being laughed at'. Humour: International Journal of Humour Research 22, pp. 145-163. 
Proyer, R. T., \& Ruch, W. (2009b). 'Intelligence and gelotophobia: The relations of selfestimated and psychometrically measured intelligence to the fear of being laughed at'. Humour: International Journal of Humour Research 22, pp. 165-181.

Proyer, R. T., Ruch, W. \& Chen, G.-H. (2012). 'Gelotophobia: Life satisfaction and happiness across cultures'. Humour: International Journal of Humour Research 25, pp. 23-40.

Proyer, R. T., Wellenzohn, S., \& Ruch, W. (2014). 'Character and dealing with laughter: The relation of self- and peer-reported strengths of character with gelotophobia, gelotophilia, and katagelasticism'. The Journal of Psychology: Interdisciplinary and Applied 148, pp. 113-132.

Ruch, W. (2004). 'Humour (playfulness)', in Peterson, C.P. \& Seligman, M.E.P. (eds.), Character strengths and virtues: A handbook and classification, New York: Oxford University Press, pp. 583-598.

Ruch, W. (2008). 'The psychology of humour', in Raskin, V. (ed.), A Primer of Humour, Berlin, Germany: Mouton de Gruyter, pp. 17-100.

Ruch, W., Beermann, U., \& Proyer, R. T. (2009). 'Investigating the humour of gelotophobes: Does feeling ridiculous equal being humorless?' Humour: International Journal of Humour Research 22, pp. 111-143.

Ruch, W., \& Proyer, R. T. (2008a). 'The fear of being laughed at: Individual and group differences in gelotophobia'. Humour: International Journal of Humour Research 21, pp. 47-67.

Ruch, W. \& Proyer, R. T. (2008b). 'Who is gelotophobic? Assessment criteria for the fear of being laughed at'. Swiss Journal of Psychology 67, pp. 19-27.

Ruch, W., \& Proyer, R. T. (2009). 'Extending the study of gelotophobia: On gelotophiles and katagelasticists'. Humour: International Journal of Humour Research 22, pp. 183-212.

Ruch, W., Proyer, R. T. \& Ventis, L. (2010). 'The relationship of teasing in childhood to the expression of gelotophobia in adults'. Psychological Test and Assessment Modeling 52, pp. 77-93.

Søbstad, F. (1993). 'Humour som ressource'. Børn og unge 47, pp. 2-4.

Stern, W. (1925). 'Grundlinien des jugendlichen Seelenlebens' [Outlines for a psychology of youth], in Küster, H. (ed.), Erziehungsprobleme der Reifezeit, Leipzig, Germany: Quelle, pp. 28-43.

Titze, M. (2009). 'Gelotophobia: The fear of being laughed at'. Humour: International Journal of Humour Research 22, pp. 27-48.

Weibel, Y. S., \& Proyer, R. T. (2012). 'Wie gehen Erwachsene mit dem Lachen und Auslachen um? Zur erinnerten Rolle von Lehrern, Familie und Gleichaltrigen' [How do adults deal with laughter and being laughed at? The remembered role of teachers, family, and peers]. Psychologie in Erziehung und Unterricht 59, pp. 81-92. 